\title{
Non-reciprocal optical reflection from a bidimensional array of subwavelength holes in a metallic film
}

\author{
Michaël Sarrazin and Jean Pol Vigneron \\ Laboratoire de Physique du Solide, \\ Facultés Universitaires Notre-Dame de la Paix, \\ rue de Bruxelles 61 , B-5000 Namur, Belgium
}

(Dated: October 28, 2018)

\begin{abstract}
Using simulations and theoretical arguments we investigate the specular reflection of a perforated gold film deposited on a glass substrate. A square lattice of cylindrical holes is assumed to produce the periodic lateral corrugation needed to hybridize the surface plasmons with radiative states. It is shown that, contrasting transmission approaches, a knowledge of the reflection on either side of the film provides separate information on the gold-vacuum surface plasmons and on the goldglass interface plasmons. Recent experimental data on a specific implementation of this system are reexamined; these show a good agreement between the measured reflections and the simulations in both directions of incident wave probes. This confirms the importance of taking into account the reflection asymmetry in the far-field assessment of surface plasmons properties.
\end{abstract}

PACS numbers: 78.20.-e, 42.79.Dj, 42.25.Bs, 42.25.Gy

In recent years, subwavelength hole arrays in metallic films have received considerable attention, mainly because of the experimental findings of Thomas Ebbesen 1 . Such systems show a particularly intriguing zeroth order optical transmission : first, the specularly transmitted beam shows an intensity higher than that of the incident beam filtered by the geometrical aperture of the holes; second, the transmitted beam develops a strong characteristic wavelength dependence ${ }^{\underline{1}}$. Recent theoretical and experimental studies have tried to clarify the detailed mechanisms involved in these phenomena 1.2.3.4.5.6.7.8. In this context, it was rapidly recognized that surface plasmons (SPs) could be a key to the understanding of the transmission measurements 1,2,3,4,5,6,7.8.

To be more precise, when the incident light illuminates the array of apertures, many diffraction orders are generated. Some of them are evanescent and exhibit a resonant coupling with SPs. They contribute to the zeroth diffraction order as a result of multiscattering and the strong exaltation of the electromagnetic field associated with resonant processes results in an enhanced transmission 1.2 .3 .4 .5 .6 .7 .8 . In a recent work ${ }^{6}$ we have suggested redefining the role of SPs in Ebbesen experiments and placing it in the required context of resonant Wood

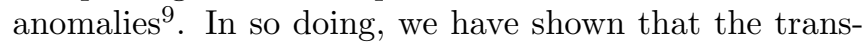
mission spectrum could be better depicted as a series of Fano profiles. These recognizable lineshapes result from the interference of non resonant transfers with resonant transfers which involve the film eigenmodes and evanescent diffraction orders. We were then able to draw attention to the fact that each transmission maximumminimum pair can be viewed as a Fano profile ${ }^{10}$. On quantitative grounds, the interplay of such an assymetric Fano profile actually dissociates the presence of a maximum or minimum in the transmission spectrum from the expected location of an eigenmode resonance ${ }^{\underline{6}}$.

It is somewhat surprising that reflection has not been as widely studied as transmission. One of our early con- tributions to this field described the theoretical reflection of an array of holes in a chromium film $\underline{\underline{6}}$ and, at about the same time, Altewischer et al reported experimental reflection studies which emphasized its non-reciprocal properties ${ }^{7}$. As is well-known, there is a significant difference between the reflection spectra for light beams coming from one side or the other side of the device. Such difference is not observed in the transmission spectra, in agreement with the reciprocity theorem 11 . The dissymmetry observed in the reflection spectra usually contains very significant information and both reflection and transmission should be thoroughly understood before a full knowledge of the optical properties of the system can be claimed ${ }^{6.7}$. The present paper proposes a theoretical investigation of the device studied by Altewischer et $\mathrm{al}^{\mathbf{l}}$. This will provide an opportunity to establish the role of SPs in these experiments and, at the same time, relate the far-field transfer coefficients to the very local SPs dynamics.

The simulations in this paper are based on a coupled modes method which combines a scattering matrix formalism with a plane wave representation of the fields $\underline{\underline{6}}$. The inherently slow convergence of this representation is here accelerated by a $\mathrm{Li}$ algorithm 12 . This technique provides a computation scheme for the amplitude and polarization $(s$ or $p$ ) of reflected and transmitted fields in any diffracted order ${ }^{6}$.

Fig 1 describes the system under study, chosen to model the samples experimentally investigated by Altewischer ${ }^{7}$. These samples can be described as a square lattice grating of circular holes perforated in a thin gold film deposited on a glass substrate. In accordance with experiments, $100 \mathrm{~nm}$ is set for the radius of each hole and the lattice parameter is taken to be $a=700 \mathrm{~nm}$. The film thickness is matched to the experimental value of $200 \mathrm{~nm}$. The gold permittivity is obtained from experimental tables 13 . The substrate slab is modelled by a semi-infinite glass medium with a refractive index of 
FIG. 1: A view of the system under study ${ }^{7}$. Transmission and reflection are calculated for zero diffraction order and normal incidence.

FIG. 2: (color online) (a) : Calculated transmission (curve marked $\mathbf{T}$ ) and reflection for zero diffraction order on the structure of Fig 1 On reflection curves, the solid (dashed) line refers to incidence from the vacuum (glass) side. The reflection nonreciprocity (difference according to incident directions) takes the form of two peaks at wavelengths $\left(1^{\prime}\right)$ and $\left(2^{\prime}\right)$ for vacuum incidence, while only one at wavelength $\left(2^{\prime}\right)$ remains in glass incidence. Other panels : (b) computed (dashed) transmission and, (c) and (d) reflections (from vacuum and glass, respectively) compared to measurements (solid) from Altewischer et $a l^{7}$. Note that experimental values are not available above $1100 \mathrm{~nm}$.

$\sqrt{\varepsilon_{s}}=1.51^{7}$. Note that the experimental device exhibited a very thin bonding layer of titanium which separates the gold film from the substrate ${ }^{7}$. The vanishing thickness of this film justifies that it will not be considered in our calculations. The linearly polarized incident light is normal to the interface. Its electric-field vector is oriented along the $[1,0]$ direction, which joins the centers of nearest-neighboring holes.

Fig 2(a) shows the specular reflection as a function of the incident wavelength in both directions of propagation, i.e. an incidence from vacuum or from glass. The transmission is also shown, with a 5-times expansion of the vertical scale ${ }^{7}$. The reflection presents strong variations near wavelengths $757 \mathrm{~nm}\left(1^{\prime}\right), 812 \mathrm{~nm}\left(2^{\prime}\right)$ and $1146 \mathrm{~nm}\left(3^{\prime}\right)$. These variations are fully consistent with experimental spectral features found at $747 \mathrm{~nm}, 810 \mathrm{~nm}$ and, presumably, at just over $1100 \mathrm{~nm}^{7}$. Fig[2(b), 2] (c), and 2 (d) reproduces the curves shown on Fig 2(a), but exposes them to a comparison with the corresponding experimental data. Despite the necessary idealization step in designing the modelled theoretical device, we note an excellent agreement in all these comparisons 7 . In particular, the relative locations of transmission maxima and reflection minima are correctly predicted. The only discrepancies which may still raise questions seem to be the weak oscillations found in all calculated spectra between $850 \mathrm{~nm}$ and $1050 \mathrm{~nm}$, not found in experiment. The spectral line shapes are reminiscent of those described for similar systems in recent computation 6 and experimental 8 reports. The difference between the reflections on both sides of the film, i.e. the reflection nonreciprocity, is evident from the comparison of Fig 2/ c) and Fig 2/d). These non-reciprocal properties of the reflection were also mentioned by Barnes et $a^{18}$ when describing their optical properties of structured metallic films.

In the present investigation the coupling between the various evanescent p-polarized diffraction orders and the surface plasmons is of prime importance. Because of the periodic corrugation of the film, the diffracted surface plasmons are sampled by the diffracted probe field at
FIG. 3: (color online) Electric field modulus spectrum for various diffraction orders for light impinging from the vacuum side (solid line) or from the glass side (dashed line) of the film. (a) Diffracted field of orders $( \pm 1,0)$ on the substrate/metal interface. (b) Diffracted field of orders $( \pm 1,0)$ at the vacuum $/$ metal interface. (c) Diffracted field of orders $( \pm 1, \pm 1)$ at the substrate/metal interface. The amplitude of the incident field is taken to be $1 \mathrm{~V} \cdot \mathrm{m}^{-1}$. (d) Absorption from the incident wave against wavelength, for incidences from the vacuum side (solid line) and from the glass side (dashed line).

many frequencies. The resonant coupling of surface plasmons to p-polarized diffraction orders at matching frequencies and surface wave vectors will lead to what will be called "resonant diffraction orders". For an isolated surface plasmon which develops on a single interface, the wavelength which characterizes each resonant diffraction order is given by ${ }^{14}$

$$
\lambda_{s p}^{u / m}(i, j)=\frac{a}{\sqrt{i^{2}+j^{2}}} \operatorname{Re} \sqrt{\frac{\varepsilon_{u} \varepsilon_{m}}{\varepsilon_{u}+\varepsilon_{m}}} .
$$

In this equation, $\lambda_{s p}=2 \pi c / \omega_{s p}$ where $\omega_{s p}$ expresses the resonant angular frequency, $a$ is the grating parameter and $(i, j)$ denotes the vector $\mathbf{g}$ of the reciprocal lattice such that $\mathbf{g}=(2 \pi / a)(i, j) . \varepsilon_{m}$ is the metal permittivity and $\varepsilon_{u}$ is either the permittivity of glass $\left(\varepsilon_{s}\right)$ or vacuum $\left(\varepsilon_{v}=1\right)$.

For the resonant orders $( \pm 1,0)$ we get at substrate/metal and vacuum/metal interfaces $\lambda_{s p}^{s / m(1,0)}=$ $1084.6 \mathrm{~nm}$ and $\lambda_{s p}^{v / m(1,0)}=720.5 \mathrm{~nm}$ respectively. Both wavelengths are indicated by vertical dashes (3) and (1) respectively in the Fig[2] and Fig[3] For the resonant orders $( \pm 1, \pm 1)$ at substrate/metal interface, we get $\lambda_{s p}^{s / m(1,1)}=787.7 \mathrm{~nm}$ indicated by dash (2). Dash (0) corresponds to the resonant orders $( \pm 2,0)$ at substrate/metal interface for $\lambda_{s p}^{s / m(2,0)}=608.1 \mathrm{~nm}$. This resonance appears in transmission as the first peak at $610 \mathrm{~nm}$.

The contrast between the reflection spectra for both incidence directions allows additional information to be obtained on processes involving specifically the surface plasmons at both interfaces ${ }^{7}$. For light arriving from the vacuum, two dips at the wavelengths $\left(1^{\prime}\right)$ and $\left(2^{\prime}\right)$ are observed. By contrast, for light arriving from the glass side a significant dip shows up at the wavelength $\left(3^{\prime}\right)$ while $\left(1^{\prime}\right)$ almost disappears. Altewischer et a ${ }^{7}$ suggested that such an asymmetry in the reflection spectra could lead to the identification of the interface on which the SPs are dominantly excited. We think that, furthermore, these results indicate that the excited resonances are mainly localized on the interface which is illuminated first and thus their effects are more easily perceived with reflection than with transmission.

To confirm and detail this interpretation, we have computed the amplitudes of main resonant diffraction orders as a function of wavelength and illumination directions. 
The field amplitude is considered here as a measure of the degree of excitation of a surface plasmon located on a specific interface. Fig 3 a shows the local amplitude of resonant orders $( \pm 1,0)$ at the substrate/metal interface. When the light crosses the substrate/metal interface at the first place, it shows a peak amplitude at the wavelength (3), predicted by Eq1 One notes a second peak at wavelength $\left(3^{\prime}\right)$ close to $(3)$, possibly related to interferences of a true resonance with a non-resonant process, as found in Fano resonances ${ }^{6}$. These peaks can easily be associated with the dip at similar wavelengths in the reflection spectrum. By contrast, when the vacuum/metal interface is illuminated first, the amplitude of these resonances collapses with the result that there is an attenuation of the associated dip in the reflection spectrum.

Fig[3b shows resonant orders $( \pm 1,0)$ on the vacuum/metal interface. Here the resonance develops at the wavelength (1) and we note the existence of two other peaks at wavelengths $\left(1^{\prime}\right)$ and $\left(2^{\prime}\right)$. It is likely that these peaks arise from a surface plasmon splitting due to the coupling of the $( \pm 1,0)$ plasmon orders at the vacuum/metal interface with the $( \pm 1, \pm 1)$ plasmon orders at the substrate/metal interface. If the vacuum/metal interface is illuminated first, amplitudes of peaks (1) and $\left(1^{\prime}\right)$ are stronger than in the opposite illumination. This explains the nonreciprocity found in the reflection spectra for wavelengths close to $\left(1^{\prime}\right)$. The spectral region close to the wavelength $\left(2^{\prime}\right)$, of the resonant order amplitude, does not significantly change when changing the propagation direction, leading to a weak nonreciprocity effect in the reflection spectrum. In Fig.3c we show the amplitude of orders $( \pm 1, \pm 1)$ at the substrate/metal interface and focus on the resonance wavelength noted (2). A major contrast is obtained, with a large field amplitude under the direct illumination of the substrate/metal interface and a much weaker amplitude under illumination from the vacuum side.

The amplitude of the resonances of orders $( \pm 1, \pm 1)$ at the substrate/metal interface and $( \pm 1,0)$ at the vacuum/metal interface are influencing each other because of the proximity of their resonance wavelengths. Indeed, the profile of the orders $( \pm 1,0)$ amplitude at vacuum/metal interface, just after the wavelength (1), can be understood as a Fano profile. Such a Fano resonance can be interpreted as resulting from the interference of the orders $( \pm 1,0)$ resonance at the vacuum/metal interface, and the orders $( \pm 1, \pm 1)$ resonance at the substrate/metal interface which then appears as a result of multiscattering of $( \pm 1,0)$ into $( \pm 1, \pm 1)$. This explains, for instance, why the wavelengths $\left(1^{\prime}\right)$ and $\left(2^{\prime}\right)$ do not strictly correspond to the resonance wavelength of the different resonant orders. Fano profiles resulting from the interference of each order as a result of multiscattering lead to a visible resonance wavelength shift 6 .

We note that this analysis of the comparison between surface plasmon features and reflection corroborate the interpretation of Altewischer et al ${ }^{7}$, from which useful information about surface plasmons can be drawn from the study of the reflection nonreciprocity.

In addition to transmission and reflection, it might be useful to investigate the loss response function for both directions of illumination. This is shown in Fig $3 \mathrm{~d}$. As light comes from the vacuum side, we observe two sharp peaks at wavelength $\left(1^{\prime}\right)$ and $\left(2^{\prime}\right)$. These peaks correspond to the surface plasmon resonance already described above. On the other hand, when the light enters through the glass side, the first peak collapses, whereas another large double peak appears at the wavelength $\left(3^{\prime}\right)$, in perfect correlation with the surface plasmon excitation. This, again, is in full agreement with the experimental results of Barnes et $\mathrm{a}^{1}{ }^{8}$ and previous computations ${ }^{6}$, confirming that both loss peaks correspond to a surface plasmon resonance. The loss line shapes can easily be correlated with the dominant features of the reflection spectra. These features indicate that, when SPs are excited, a large part of their energy is dissipated via the Joule effect, leading to lower reflection intensities. In addition, a smaller part of SPs energy is transferred to the transmitted beams as a result of multiscattering. So, as SPs are essentially excited on the interface which is encountered first, the reflection spectrum is dominated by processes associated with the losses from the plasmons carried by this interface.

As already noted by Barnes ${ }^{8}$, this is quite different for transmission, where SPs of both interfaces equally contribute to enhanced transmission whichever side is illuminated first. The interface which receives the incident beam provides strong SPs resonances. Such resonances only give a weak contribution to the transmission since the associated field is damped when propagating through the holes before providing transmitted intensity. On the opposite interface, SPs are excited by an incident light weakened by its propagation through the holes, so that SPs on the opposite interface are only weakly excited. Their contribution to the transmitted field is thus also weak. In other words, SPs of the illuminated interface are strongly excited and then damped after propagation through the holes, whereas SPs on the opposite interface are weakly excited as the incident light is first damped. The result is that SPs of both interfaces contribute to the transmission with similar magnitudes and that the choice of the direction of propagation does not influence the information content of the measured transmission.

In conclusion, the examination of several experimental studies 7.8 in the light of the interpretation scheme presented elsewhere ${ }^{6}$ reinforces the point of view expressed in this scheme by exhibiting qualitative and quantitative agreements between measured and computed light filtering spectra of a perforated gold layer covering a glass substrate. A particular effort was made to study the nonreciprocity property of the reflection. It was found that the asymmetry relative to the light propagation direction is effectively caused by the localization of SPs resonances at gold film surfaces and that this can be exploited to investigate separately the gold/glass and gold/vacuum plasmon coupling to radiative waves. The transfer func- 
tions computed in detail in this paper agree well with the quoted experimental data, available to us at the time of writing. One of the outcomes of these experiments is the role played by the corrugated-surface plasmons in the build up of the light transport process. In order to shed some light on this issue, the present work also looked into the theoretical value of the excited field at the location of the metal/vacuum and metal/glass interfaces and determined the degree of excitation of the interface plasmons. The strong correlation found between the plasmon amplitude and the reflection variations shows unambiguously the role played by the metal surface collective excitations in the light transfer process. This conclusion suggests that the experiments could be taken some steps further by considering complementary measurements, at least on the outer vacuum/gold surface, of the far field in reflectance experiments and the near field with the scanning near-field optical microscope. A correlation between these separate investigations should be highly instrumental in elucidating the mechanisms of the light transfer processes.

\section{Acknowledgments}

We thank M.P. van Exter and E. Altewischer for enlightening advice and for making extensive experimental data available to us during the preparation of this work and P. Kelly for his advice on the manuscript.

We acknowledge the use of the Namur Interuniversity Scientific Computing Facility (Namur-ISCF), a joint project between the Belgian National Fund for Scientific Research (FNRS), and the Facultés Universitaires NotreDame de la Paix (FUNDP).

This work was partially supported by the EU BelgianFrench INTERREG III project "PREMIO", the EU5 Centre of Excellence ICAI-CT-2000-70029 and the InterUniversity Attraction Pole (IUAP P5/1) on "Quantumsize effects in nanostructured materials" of the Belgian Office for Scientific, Technical, and Cultural Affairs.
* Electronic address: michael.sarrazin@fundp.ac.be

1 T.W. Ebbesen, H.J. Lezec, H.F. Ghaemi, T. Thio, P.A. Wolff, Nature (London) 391, 667 (1998).

2 H.F. Ghaemi, T. Thio, D.E. Grupp, T.W. Ebbesen, H.J. Lezec, Phys. Rev. B 58, 6779 (1998).

3 E. Popov, M. Nevière, S. Enoch, R. Reinisch, Phys. Rev. B 62, 16100 (2000).

${ }^{4}$ L. Martin-Moreno, F.J. Garcia-Vidal, H.J. Lezec, K.M. Pellerin, T. Thio, J.B. Pendry, and T.W. Ebbesen, Phys. Rev. Lett. 86, 1114 (2001).

5 A. Degiron, H.J. Lezec, W.L. Barnes, T.W. Ebbesen, Appl. Phys. Lett. 81, 4327 (2002).

6 M. Sarrazin, J.-P. Vigneron, J.-M. Vigoureux, Phys. Rev. B 67, 085415 (2003).

7 E. Altewischer, M.P. van Exter, J.P. Woerdman, Optics
Letters 28, 1906 (2003).

8 W.L. Barnes, W.A. Murray, J. Dintinger, E. Devaux, and T.W. Ebbesen, Phys. Rev. Lett. 92, 107401 (2004).

${ }^{9}$ R. Wood, Phys. Rev. 48, 928 (1935).

10 V. Fano, Ann. Phys. 32, 393 (1938).

11 R. Petit, Electromagnetic Theory of Gratings, vol. 22 of Topics in current Physics (Springer Verlag, 1980).

12 L. Li, JOSA A 14, 2758 (1997).

13 D.W. Lynch, W.R. Hunter, in Handbook of Optical Constants of Solids, edited by E. D. Palik (Academic Press, Inc., 1991), vol. 2.

14 H. Raether, in Springer Tracts in Modern Physics, edited by G. Hohler, E.A. Nickisch (Springer, Berlin, 1988), vol. 111. 


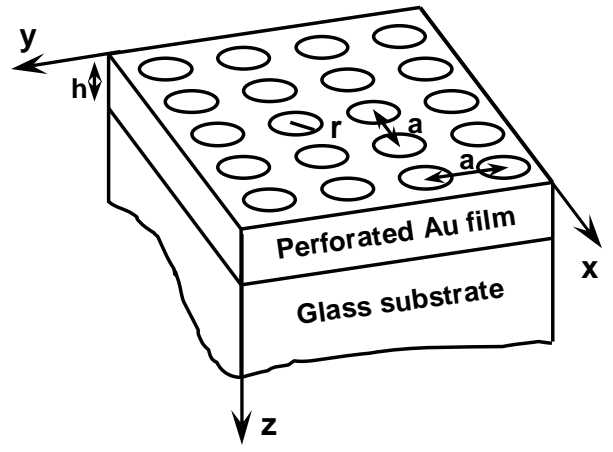



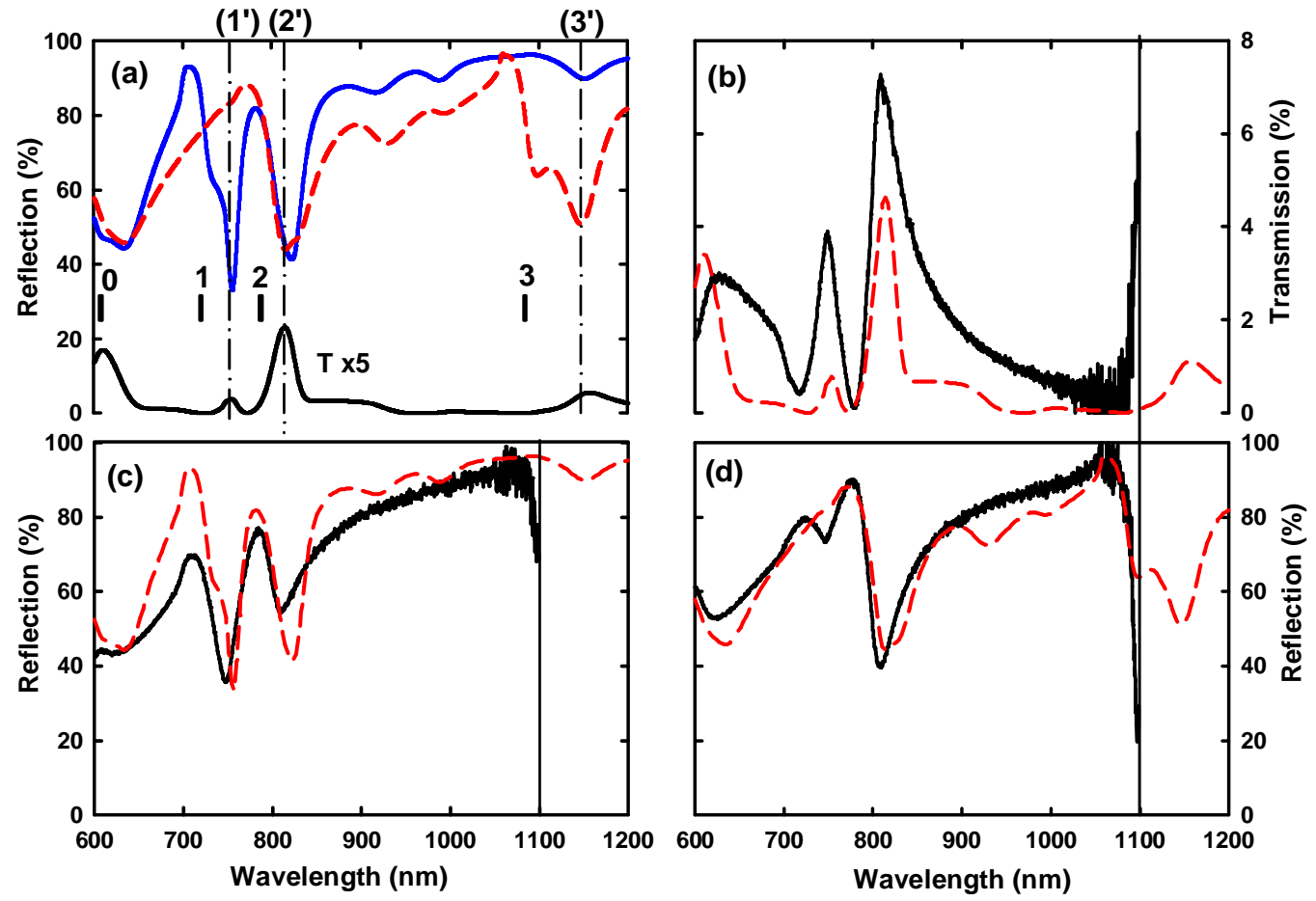


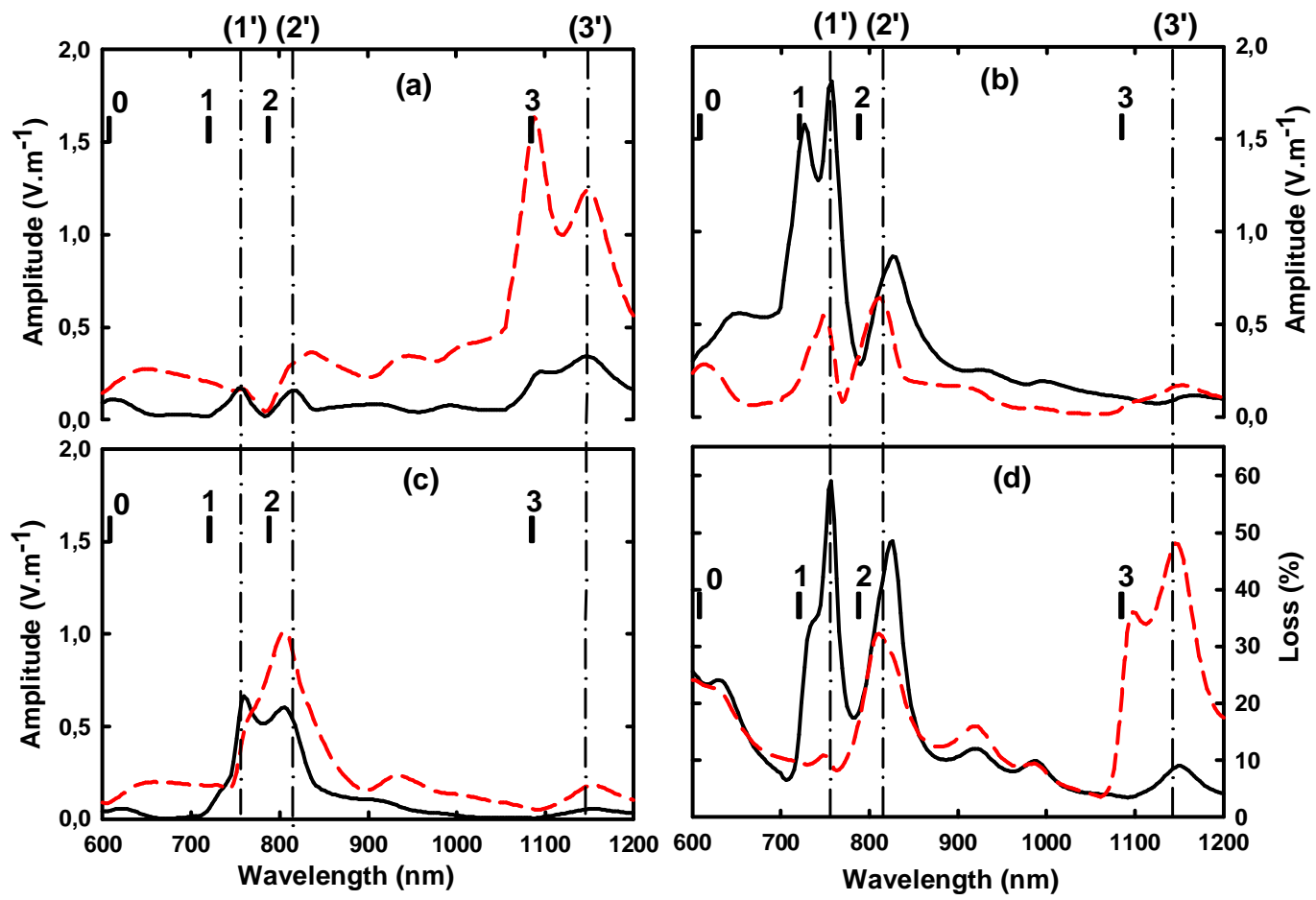

